\title{
Quaderni
}

QUADERNI Communication, technologies, pouvoir

73 | Automne 2010

La métropole parisienne entre récits, paroles et échanges

\section{La métropole parisienne et ses récits : du projet de territoire à une possible identité narrative dialoguée?}

Dominique Pagès

\section{(2) OpenEdition}

\section{Journals}

Édition électronique

URL : http://journals.openedition.org/quaderni/441

DOI : 10.4000/quaderni.441

ISSN : 2105-2956

Éditeur

Les éditions de la Maison des sciences de l'Homme

Édition imprimée

Date de publication : 5 octobre 2010

Pagination : 9-24

\section{Référence électronique}

Dominique Pagès, «La métropole parisienne et ses récits : du projet de territoire à une possible

identité narrative dialoguée? ", Quaderni [En ligne], 73 | Automne 2010, mis en ligne le 05 octobre 2012, consulté le 01 mai 2019. URL : http://journals.openedition.org/quaderni/441 ; DOI : 10.4000/ quaderni.441 


\section{$D$ ossier}

la métropole parisienne et ses récits : du projet de territoire à

une possible identité narrative dialoguée?

\author{
Dominique \\ Pagès \\ Maître de Conférences \\ CELSA, GRIPIC, \\ Université Paris Sorbonne
}

Les métropoles mondiales et internationales, territoires emblématiques de la globalisation économique et de la mondialisation culturelle, sont aussi celles d'expériences sensibles au quotidien, de vécus souvent inédits que les sciences sociales tentent tant bien que mal d'analyser en renouvelant les regards et les paradigmes.

Ces métropoles « d'un nouveau type » semblent vouloir réinventer les formes, les lieux et les temps du vivre ensemble : en relativisant les périmètres institutionnels et les frontières établies, en rendant proche l'ailleurs et l'ici insolite, en intégrant la différence, en proposant de nouvelles relations entre la nature et la ville (les territoires élargis et les grands paysages). Ces tensions, entre deux échelles (celle du local, voire du micro-local et celle du mondial), entre deux états (minéral et végétal) ou encore entre deux regards (le spectaculaire et l'ordinaire) se traduisent au fil des innombrables discours et récits dont elles sont l'objet.

Les métropoles et mégalopoles du $\mathrm{XXI}^{\mathrm{e}}$ siècle cherchent donc non seulement leur forme mais aussi leur langage et leur définition. Il s'agit de sortir théoriquement (mais sans les rejeter) des approches reçues en héritage : celle de la « Ville radiocentrique » et de son modèle de croissance, celle de la «Ville Écologique » et de sa célébration du péri-urbain, celle de la «Ville liquide » et de ses flux (inspirée par les réflexions de Zygmunt Bauman sur la vie et la société liquides), celle de la « Mégalopole du pire » évoquée par Mike Davis ou encore celle de « la Ville globale » (siège emblématique des multinationales, des banques, des bourses, des services, déconnecté de son environnement proche, dont le skyline 
apparaît comme une signature) mise en question par Saskia Sassen.

Mais il s'agit aussi pour chaque métropole d'affirmer dans cet apparent jeu de concurrence mondiale une vision attractive du « vivre ensemble post-urbain » qu'elle ajuste afin d'attirer tout à la fois investisseurs et « cerveaux », touristes et nouveaux habitants.

\section{La singularité du cas de la Métropole de Paris}

Après avoir été un enjeu technique puis de politique locale, la métropolisation de Paris, en constitution théorique, politique et culturelle, semble vouloir s'affirmer progressivement comme un enjeu civilisationnel dépassant tous les clivages partisans. Ainsi, depuis deux années, au fil des médias, le projet du Grand Paris s'est énoncé comme une question centrale pour le devenir de la société et de la culture françaises. La question du processus de métropolisation de Paris a fait l'objet de prises de position contrastées ${ }^{1}$, d'une couverture et d'un traitement médiatiques superlatifs. Une conception française du processus de métropolisation, cherchant sans le dire une exemplarité, s'est racontée au monde et surtout s'est mise en image.

Il s'agira ici non pas d'analyser ce processus de métropolisation en soi mais d'étudier les trois principaux récits qui le traduisent et le légitiment : celui de la Mairie de Paris (via sa délégation Paris Métropole et l'action de l'élu mandaté mais aussi via le Syndicat Mixte Paris Métropole, créé à partir de la Conférence Métropolitaine), celui de l'État et enfin celui, potentiel plus que véritablement actuel, de « la Métropole des individus $»^{2}$.
Récits nécessairement contrastés dans leur vision du territoire, dans leur mode d'énonciation de la dynamique de développement, dans leur tonalité affective et leur prise en compte des habitants. Récits portés par des voix qui différent tant dans le propos, le ton que la forme de leur légitimation.

Nous n'avons pas pris ici en compte la prise de position régionale car, malgré la richesse des discours et des études, la profusion des déclarations, il nous semble que le récit ne soit pas encore véritablement constitué et surtout lisible au-delà des sphères expertes et locales.

\section{Récits plus que discours?}

Les grandes métropoles sont beaucoup plus que des agglomérations ou des villes «élargies », elles sont une réécriture en profondeur de nos modes d'habiter, de circuler, d'appartenir à un territoire. Elles sont donc affaire de représentations et de symboles sans cesse renouvelés. Émanations de villes emblématiques mais en rupture avec leur géographie, leur passé (qu'il soit muséal, industriel, de services), elles se constituent en nouvelles légendes territoriales devant attirer à la fois l'attention mondiale et susciter la fierté locale. Pour cela, elles sortent d'une approche strictement publicitaire et d'image de marque (le classique " marketing territorial ») pour s'affirmer symboliquement comme de nouvelles mythologies contemporaines au fil d'une série d'évènements et d'un enchaînement d'actions qui les produisent en continu : celles des politiques, celles des urbanistes, des architectes et des aménageurs, celles des acteurs confusément regroupés sous l'appellation de « société civile» (associations et autres médiateurs, habitants). 
Elles se déploient dans le temps et dans l'espace, se configurent et reconfigurent au fil de manifestations majeures, se transforment « sous nos yeux » pour proposer de «nouveaux mondes possibles». Les métropoles nous parlent de leur conversion, trament progressivement leur identité narrative et se mettent en intrigue.

Mais cette mise en récit est moins univoque qu'il n'y paraît. Ainsi pour le cas de la Métropole de Paris, l'écriture du processus de métropolisation reste contradictoire : l'écriture patiente du projet « Paris Métropole » de la Ville de Paris, porté depuis 2001 par un adjoint au Maire, Pierre Mansat, et sa délégation, a du faire front et s'adapter à l'écriture épique d'un nouveau mythe Métropolitain, « la Métropole de l'après Kyoto », par Nicolas Sarkozy et aujourd'hui semble vouloir chercher, de manière encore indécise, le relais des récits ordinaires pour se redéployer. En effet, il semble que l'émergence diffuse de paroles « ordinaires » d'une diversité d'acteurs (associations, collectifs d'artistes, altermédias mais aussi simples habitants) s'intéressant au devenir de Paris, marque une étape dans le processus de métropolisation et puisse instaurer une fertile tension entre « récit spectaculaire » et « récit vernaculaire $»^{3}$. L'importance croissante des médias numériques conforte ce mouvement.

\section{Paris Métropole, un projet de territoire}

Depuis 2001, la démarche de métropolisation s'est instituée par le biais de la création au sein de la Mairie de Paris d'une délégation dédiée (nommée en 2001 «Relations de Paris avec les collectivités territoriales d'Île de France » puis renommée, en 2008, en termes politiques
« Paris Métropole » et en termes administratifs « Délégation générale à Paris Métropole et aux coopérations interterritoriales »). Le projet n'était pas neuf ${ }^{4}$ mais il trouva là une assise et un soutien politiques nécessaires à sa théorisation, sa promotion et sa mise en œuvre. La logique et la démarche retenues furent celles d'un développement territorial de grande ampleur : il s'agissait de transformer progressivement, par la voie du dialogue, les relations entre Paris et sa banlieue, de faire évoluer les points de vue de Paris sur sa banlieue et de la banlieue sur Paris ainsi que les formes et méthodes de collaboration.

Faire apparaître, au fil d'un diagnostic partagé (pénurie de logements, mobilité résidentielle compromise, dérive pavillonnaire, étalement urbain nuisible, mal vivre des cités, mitage de nos paysages, etc.) puis d'incessantes discussions entre élus et experts, une dynamique métropolitaine inédite : ce fut et cela reste le moteur de cette interpellation collective des acteurs du territoire de l'agglomération parisienne.

Discrètement, le refus de persister dans la gestion des territoires selon leurs délimitations institutionnelles, le souci de mettre en œuvre un processus méthodique de rééquilibrage économique et social du territoire et donc la volonté de faire émerger une nouvelle forme d'organisation territoriale sont devenus pour les élus du centre et de la périphérie des enjeux partageables. La démarche d'apprentissage progressif, itérative, fondée sur le dialogue et le débat a en effet permis de forger une rationalité commune, de faciliter les coopérations entre les acteurs, de parvenir à la création d'une conférence métropolitaine puis d'un Syndicat mixte (Paris Métropole). 


\section{La construction d'un sens commun}

Cette dynamique participative fut donc portée par un adjoint, Pierre Mansat, élu communiste à l'époque, qui joua tour à tour le rôle d'ambassadeur, d'évangélisateur et de médiateur mais sans jamais avoir véritablement les moyens statutaires et logistiques pour installer un leadership et donc une voix au-delà des sphères expertes. Sa quête d'un consensus politique local, son dialogue avec des élus de tous bords, l'effort de sa délégation à organiser une concertation avec tous les acteurs concernés, à la traduire par une politique de communication relativement classique et, pendant longtemps, confidentielle, permirent de substituer à l'approche technique l'écriture politique et citoyenne d'une métropolisation.

L'objectif communicationnel était à la fois d'installer une vision consensuelle autour d'une métropole de l'intégration et de la solidarité (sa définition évolutive ayant été confiée aux chercheurs - géographes, urbanistes, politistes) et de permettre le débat en continu entre les élus, les experts et les opérateurs. Pour illustrer cette communication du projet, citons quelques moments et actes forts :

- l'invitation le 5 décembre 2001par Bertrand Delanoë des élus de la première couronne à la Maison de la RATP, qui donnera lieu à une publication ; - l'exposition en 2002 à l'Arsenal « Territoires partagés, l'archipel métropolitain » qui cherchait à montrer une métropole active regroupant Paris et les communes de l'agglomération dans un destin commun ;

- la conférence métropolitaine, lieu d'échanges et de débats, d'analyses et de concertation entre les élus qui s'est réunie pour la première fois à
Vanves le 7 juillet 2006 ; elle fut pensée comme un espace « commun à tous », permettant de faire se confronter des idéologies et des représentations différentes, des opinions et des engagements contradictoires ; elle portait d'emblée cette espérance d'un « orchestre métropolitain »;

- le cycle de rencontres, débats et ateliers, d'évènements artistiques « Entre Villes et Métropoles » (organisé en partenariat avec la Maison de l'Europe et la Ville de Paris en 2008). Celui-ci sera suivi par d'autres évènements intégrant progressivement les citoyens aux échanges.

Des publications accompagnèrent donc ce mouvement mais en se cantonnant au statut de documents professionnels et relativement experts : des cartographies tentèrent de visualiser les possibles périmètres de la métropole à moyen et long terme (de 200 à 400 communes possibles pour 2020); des ouvrages de qualité furent publiés notamment via $1^{\prime} \mathrm{APUR}^{5}$; des études historiques cherchant à fouiller la mémoire des rapports « Paris et Banlieues » furent commandées et éditées ; une lettre de coopération territoriale créée dès 2001, Extra Muros, connut un succès évident jusqu'en 2009 (son dernier numéro Hors série est en cours de publication); des supports évènementiels traduisirent méthodiquement l'avancée du projet ; dès 2002, une rubrique conséquente (bien qu'assez difficile à atteindre) fut créée sur le site de la Mairie de Paris : intitulée tout d'abord «Coopération Paris Banlieue » et voulant « rendre intelligible et concrète la nouvelle démarche de partenariat engagée entre Paris et les Collectivités d'Ile de France », elle fut donc renommée « Paris Métropole » en 2008.

Mais ces actions de communication précises et 
rigoureuses restèrent longtemps invisibles pour le citoyen, malgré l'effort de quelques rares médias à les commenter (ainsi la rubrique du Nouvel $O b$ servateur consacrée à ce sujet mais qui disparut en 2008). Sans doute, la place d'intermédiaire donnée à Pierre Mansat et celle attentiste de Bertrand Delanoë qui ne chercha pas à s'imposer sur ce sujet en tant que leader ne permirent pas de donner au projet de Paris Métropole une plus grande présence médiatique.

\section{Un syndicat naissant inaudible pour le grand public?}

Ce fut l'entrée en scène et la mise en spectacle du projet Grand Paris qui obligèrent la délégation à sortir de sa réserve communicationnelle et à passer du discours explicatif et collaboratif au récit collégial et institutionnalisé. Le lancement du site du Syndicat Paris Métropole (créé le 10 juin 2009) ${ }^{6}$ et la présentation aux médias de son logo et de ses thématiques de réflexion (le 17 décembre 2009 au Tapis Rouge), voulurent marquer une nouvelle étape communicationnelle face à la machine médiatique mise en œuvre autour du « Grand Paris ».

Plus récemment le 23 puis le 31 avril 2010, le Syndicat sembla vouloir plus directement encore construire la riposte en relançant l'idée « pragmatique » des « 100 - puis 110 - appels à initiatives» (idée lancée dès février 2009 par la conférence Métropolitaine) faisant ainsi écho aux « 1000 projets » annoncés par Nicolas Sarkozy ${ }^{7}$. Le Syndicat déclara au nom des 110 collectivités adhérentes vouloir «être le promoteur de la participation des citoyens à une histoire, un avenir, une stratégie dont les collectivités refusent qu'ils se fassent sans eux ». L'appel ne va pas sans rappeler les méthodologies des IBA allemandes, consistant à mettre en pot commun des initiatives émergeant du terrain pour être développées collectivement (que celles-ci portent sur des actions dans l'espace public, des initiatives populaires, des évènements culturels).

Mais, de nouveau, faute sans doute d'un leardership reconnu et d'une personnalité emblématique pour donner toute sa voix à cette initiative, cette action a mis du temps à être véritablement relayée par les médias. Dans sa première année d'existence, il a certainement manqué au Syndicat, qui certes se constituait sans les moyens de ses ambitions, une voix forte et claire, reconnue pour conforter la présence médiatique du projet. Pas de vision métropolitaine sans un narrateur emblématique?

\section{Grand Paris, un récit épique}

La mise à l'agenda présidentiel du processus de métropolisation de Paris et surtout la spectacularisation de son écriture politique furent donc vécues comme des déflagrations : dès le 26 mai 2007, Nicolas Sarkozy, invité à l'Hôtel de Ville de Paris en tant que nouveau Président de la République, déclara son intention de renouer avec l'ambitieuse tradition française d'aménagement de la Capitale dans le sillage d'Haussman et de Delouvrier. Avec la déclaration de Roissy, en juin 2007, relayée par tous les médias, commença le feuilleton médiatique du Grand Paris dont le grand public suit tant bien que mal les épisodes. La création, en avril 2008, d'un Secrétariat d'État du développement de la région Capitale confiée à Christian Blanc fut un jalon essentiel, à l'origine d'un repositionnement de l'ensemble des protagonistes. 
Une mise en intrigue d'un territoire en devenir

Avec le Grand Paris, Nicolas Sarkozy construit un récit tenant en haleine une communauté élargie à l'échelle nationale et internationale : il s'est d'emblée adressé « au peuple de France », usant de tous les subterfuges de la narration; son récit recentralisateur a su dramatiser les enjeux (faire front à la mondialisation et à la crise, assumer positivement les enjeux du développement durable), se faire lyrique (« asseoir le statut de ville monde de la capitale par son rayonnement international») et visionnaire ( « un jour on ne parlera plus de Grand Paris mais de Paris tout simplement»).

Son récit, qui tend à occulter tous les autres récits existants, est à la fois un récit de fondation et un récit épique : au fil de ses déclarations et de la traduction plurimédiatique du projet, il cherche à raconter à tous un "nouveau monde commun », à proposer un autre monde possible, notamment sur un mode utopique, mais aussi à se faire le héros de cette quête civilisationnelle.

Le récit est une pratique d'élaboration du sens : celui-ci nous parle de la refondation d'une Ville Capitale, exaltant son passé glorieux mais dépassé (La Ville Lumière), ses luttes actuelles (son engorgement, sa faiblesse concurrentielle, le contexte mondial hostile) et son futur lumineux (la Métropole de l'après Kyoto). Il s'agit de faire sortir Paris de son ornière et de chercher une issue exemplaire, et cela en prenant à témoin l'opinion nationale et internationale.

Récit de fondation donc sous les auspices d'un prestigieux héros, comme la Renaissance ita- lienne sut en produire, et que l'on peut analyser par le biais d'outils méthodologiques comme le « schéma narratif » (qui s'attache à la chronologie) et le « schéma actantiel » (qui s'attache aux actions des personnages).

Ainsi, il y a bien une situation initiale (la tension Paris/banlieue, le déclin et l'engorgement de Paris, l'épuisement de son modèle urbain), une ou plutôt des perturbations (la mondialisation, la concurrence des Villes Monde, les risques écologiques et autres mises à l'épreuve du territoire), des actions à mener et des réactions face à l'adversité (l'action trop lente de la Mairie de Paris, sa vision pas assez réaliste, etc.) qui correspondent aux prouesses du héros (Nicolas Sarkozy). Ainsi, il y a bien des rivaux (dont la bravoure plus que l'action est reconnue), une résolution (notamment la consultation des équipes réunies par de grands architectes autour du Grand Paris et le projet du Grand 8 de Christian Blanc) et puis, enfin, un nouvel équilibre annoncé (La Métropole Durable).

De même, le récit métropolitain de Nicolas Sarkozy peut s'analyser plus précisément à travers le rôle et les relations entre ses protagonistes et actants. Il y a le héros qui doit ouvrir une voie face à l'adversité, surmonter les embûches, s'imposer comme le grand rassembleur assumant les enjeux de rupture (plus que de transformation, notamment dans ses premiers discours); il y a les rivaux que l'on affronte dans un jeu de joutes et de ripostes successives (fortement relayé par les médias) mais avec lesquels on peut s'allier dans cette perspective de transformation commune; il y a les experts qui apportent leur aide avant tout symbolique (les architectes et leurs équipes). 


\section{Une épopée visionnaire?}

Enfin, ce récit sur le long terme fait vibrer certaines cordes essentielles de l'identité humaine : entre 2008 et 2009, Nicolas Sarkozy a joué sur l'émotionnel, le sensationnel, l'enchantement, la force suggestive, via une événementialisation médiatique de grande ampleur de sa parole et de ses décisions. «Le Grand Paris », c’est avant tout un spectacle qui impressionne, qui veut marquer les esprits, rendre compte de l'action prophétique d'un homme qui propose une vision claire de la situation (même si souvent sans nuance), qui développe l'envie d'un changement d'avenir en transformant l'attente en puissance, qui remet en cause un ordre ancien défini comme une impasse. Le « Grand Paris », c'est l'irruption d'un mouvement charismatique qui veut être le moment de transition entre un avant et un après, c'est un récit d'envergure dont la dimension fondatrice veut marquer la culture d'un peuple, quitte à préférer la vraisemblance de la vision à la vérité du territoire.

En prenant cette posture en surplomb («se projeter dans la civilisation urbaine de l'après Kyoto »), en adoptant cette attitude de condescendance (se porter au secours de Paris, qu'il faudrait simplement réinventer), en utilisant toutes les formes de l'hyperbole, Nicolas Sarkozy conforte non seulement l'approche recentralisatrice et protectrice du chef charismatique, mais il actualise aussi différentes mythologies : celle d'une terre promise, d'un refuge du bonheur, d'un territoire de la réconciliation avec la nature.

En donnant vie et substance au « Grand Paris », il construit une épopée visionnaire : celle de la transfiguration d'un Paris épuisé, muséifié, « en retard », celle d'un homme d'envergure exaltant un idéal collectif à force d'hyperboles, d'antithèses, suggérant la grandeur, l'intensité et la force civilisationnelle d'une vision. Mais au-delà de sa clarté et de sa force performative, ce récit ne tend pas moins à atomiser les autres récits.

\section{La fabrique d'un mythe}

Plus concrètement, la consultation lancée au printemps 2008 et l'exposition à laquelle elle donna lieu à la Cité de l'Architecture et du Patrimoine dès le mois d'avril 2009 furent le point d'orgue de cette stratégie de communication. L'avenir urbain d'une grande métropole mondiale fut donc l'objet d'une consultation et non d'un concours : ce fut une première. 10 équipes pluridisciplinaires furent sélectionnées ${ }^{8} .500$ personnes planchèrent pendant plusieurs mois sur la définition d'une « Métropole Parisienne Post Kyoto » et l'opération déboucha sur une diversité de scénarios et de propositions. Chaque équipe, avec son style, son ton, a présenté son diagnostic et ses pistes de solution (plus ou moins abouties) mais aussi son « dessein » (plus ou moins utopique, concret, réaliste, poétique). Des centaines de projets ont été effleurés, correspondant à des milliers de kilomètres à repenser dans leurs usages.

L'opération fut spectaculaire, le succès populaire au rendez-vous, l'architecture renouant avec le politique mais au risque du spectacle et de l'instrumentalisation de l'un par l'autre. Certes un consensus apparent semble s'y être construit autour d'une profusion d'images donnant à voir un possible territoire métropolitain (pouvant aller jusqu'au Havre, Grumbach), de textes le 
commentant parfois jusqu'au poème (Castro), de croquis chatoyants à forte portée métaphorique, nous sortant de Paris, du Paris historique pour nous proposer une métropole hybride aux multiples visages : la «Métropole Polycentrique» (Rogers Stirk Harbour and Partners), la « Ville poreuse » (Secchi et Vigaro), la «Ville légère et réversible » (Geipel et Andi), le "Grand Paris solidaire et naturellement solidaire » (Castro)... Mais au final, malgré l'annonce de la création de l'AIGP (Atelier International du Grand Paris, sorte d'agence d'urbanisme métropolitaine, instance de conception et de coordination) qui doit travailler autour des pistes acquises et ainsi passer du récit à l'action, la définition de la Métropole reste en suspens tout comme sa réalisation. Le chœur des architectes mécontents s'est fait entendre depuis et a pointé les défaillances d'un récit performatif?.

\section{Un récit de marque?}

Au-delà du récit de Nicolas Sarkozy, le projet du Grand Paris s'est affirmé dans l'espace public avec les moyens d'une marque. Un lancement de marque qui est passé par des discours d'annonce diffusés par une diversité de médias publics et marchands (la web TV, le site citoyen, l'exposition, la radio, les retombées presse...), valorisant les attributs de cette marque territoriale. En deçà de l'épopée, le Grand Paris a intégré les indicateurs qui fondent les palmarès et les labels dont les grandes villes font aujourd'hui l'objet : «European cities of culture », «Creative Cities », «Metropole of design », labels verts, etc. Les métropoles sont de plus en plus soumises à des classements : ainsi le CBI (City Brand Index, publié par GMI et Simon Anholt) est devenu le premier baromètre mesurant les images de marque de villes internationales.

Ces stratégies utilisent la puissance évocatrice du récit et de la narrativité (et notamment du story stelling) pour promouvoir les vertus d'un territoire et en renforcer l'image. Elles spectacularisent les villes, cherchent à en soigner la réputation, en content le cadre de vie, nécessairement convivial et sécurisé, l'ambiance nécessairement cosmopolite et polysensorielle. Derrière le récit épique semble donc se profiler un récit marchand clés en main, saturé d'images prometteuses et d'incitations à la consommation d'espaces. Mais une métropole ne peut se résumer en un spectacle, en une méthode de management ou en un récit univoque. Elle ne peut se limiter à une réputation formatée, négatrice de la profondeur politique et culturelle du processus de métropolisation. Pour se constituer en tant que telle, il lui faut échapper à nombre de tentations actuelles, celle de la Ville Globale (un nœud dans un archipel mondial), celle de la Ville liquide (un territoire parcouru par les flux, sans limite), celle de la « Data Ville» (un territoire résultant d'une convergence de données).

La métropole ne peut se faire à partir du passage en force d'un homme politique aussi puissant ou entreprenant soit-il. Si Nicolas Sarkozy a su ouvrir une voie irréversible, s'il a permis, via son récit et surtout les actions que celui-ci annonçait, d'accélérer le processus de métropolisation, il ne peut continuer sur ce registre épique et flamboyant.

Il s'agit bien à cette étape du processus de prendre en compte la possible émergence d'une 
communauté métropolitaine non plus subie mais active, une communauté de sens, de solidarités, de vécus apte à construire sa propre identité narrative. Les citoyens et la diversité de leurs formes d'association, les habitants et les visiteurs et leurs relations effectives au projet métropolitain restent encore un impensé des projets métropolitains : si on les évoque, les interpelle abstraitement, les dispositifs permettant d'enregistrer et d'intégrer leurs points de vue sur la métropolisation restent sommaires, voire des leurres (ainsi le site « Mon Grand Paris » lancé fin 2009 - voir plus loin).

Les récits proposés, s'ils ont voulu raconter à leur façon un bien commun en constitution, n'ont guère envisagé jusqu'ici les conditions concrètes de leur appropriation active : ils se sont voulus informatifs ou enchanteurs, légitimant ou séducteurs mais sans penser véritablement la diversité des publics qu'ils visaient. Ces récits n'ont atteint qu'une infime partie de la population métropolitaine. Comment l'habitant peut-il penser la métropolisation à partir de ces récits contradictoires et sans autorité claire pour lui en expliquer l'incidence sur son quotidien?

La métropole, c'est un ensemble complexe de territoires hétérogènes et $d$ 'habitants aux profils et aux vécus profondément contrastés : de l'habitant péri-urbain à celui des banlieues sensibles, du jeune étudiant sillonnant son territoire via les transports en commun à l'automobiliste aux déplacements routinisés et de plus en plus difficiles. Pour être compris et discuté, le processus de métropolisation qui vise à la redéfinition d'un territoire élargi et d'un vivre ensemble doit intégrer cette diversité, l'assumer sans chercher à la simplifier, à la réduire ou à l'endiguer.

\section{La « métropole des individus » et ses nécessai- res intermédiations}

L'émergence d'une conscience et d'une opinion publique métropolitaines ne peut se contenter des seuls récits « venus d'en haut ». La métropole n'adviendra que dans la tension entre ces récits et ceux des gens qui y vivent, entre le récit spectaculaire en surplomb et le récit ordinaire ou 《 vernaculaire ${ }^{10}$. La participation des habitants à la fabrique des territoires est une vieille question mais qui se renouvelle avec le cas singulier des métropoles : ce sont de grands territoires composites pouvant entrer en contradiction avec les intérêts locaux, fondés sur une réorganisation profonde du territoire et des mobilités qui s'y déploient.

On anticipe bien le difficile passage du récit utopique ou du récit épique à leur concrétisation : la métropolisation va affecter nos modes d'habiter, de parcourir et de vivre nos territoires du quotidien, parfois superficiellement, parfois en profondeur; elle va changer nos représentations mais aussi nos pratiques du territoire. Pour la rendre possible et acceptable, la prise en compte des habitants et de leurs paroles, de leurs usages et de leurs expériences devient incontournable.

Il importe de dépasser la fascination du « no limit » pour reterritorialiser le projet en suscitant de nouveaux points de vue intermédiaires (ceux notamment des élus locaux et des associations) et, progressivement, en facilitant la parole effective des habitants, en tenant compte de leurs vécus mais aussi de leur expertise.

Depuis deux années une diversité d'initiatives a 
ouvert la voie à ce que l'on nommera provisoirement « le troisième récit » : un récit local qui tente de redonner de l'épaisseur au projet, de sortir des visions en surplomb et des cartographies spectaculaires, de questionner les totems et les imageries des architectes.

\section{Des médias aux médiations artistiques}

Des médias indépendants (du moins a priori) ont récemment questionné l'émergence du Grand Paris à partir d'une prise en compte du citoyen et de l'habitant : citons ainsi Metropolis « magazine grand format de la ville ouverte de demain » dont le $\mathrm{n}^{\circ} 0$ fut lancé en juillet 2009 avant de se reconvertir en Megalopolis, « le magazine du Très Grand Paris » $\left(\mathrm{n}^{\circ} 0\right.$ paru en novembre 2009, magazine mensuel de 48 pages) - puis lancement du site Megalopolismag.com en février 2010, visant à « accompagner l'émergence du Grand Paris et à créer une communauté métropolitaine ». Les porteurs de ce projet sont 10 étudiants de Sciences $\mathrm{Po}^{11}$ voulant aller « au plus près du terrain » pour envisager la perception du projet par les habitants. L'initiative qui semble rencontrer un certain succès a ouvert la voie à une médiatisation plus ouverte sur la réalité des territoires et sur les attentes et peurs liées au « Grand Paris ».

Des artistes investissent progressivement la question métropolitaine avec un regard et des formes d'écriture renouvelés, cherchant à sortir des stéréotypes et clichés métropolitains en place. Les métropoles mondiales sont en effet culturellement et artistiquement chargées d'un imaginaire codifié : le cinéma a depuis longtemps envisagé ces territoires en termes soit de «Villes lumière » (voir le récent Paris Je t'aime réalisé par «les plus grands réalisateurs du cinéma mondial »), de « machines de fer et de verre » (de Métropolis à Blade Runner), de « Jungle cosmopolite » (le Training day d'Antoine Fuqua), de « labyrinthe sous contrôle » (la Trilogie Jason Bourne de Doug Liman puis Paul Greengrass, ou encore Nowhere de Gregg Araki) et plus encore de «Villes catastrophes » (innombrables mises en scène de la fin de New York ou de Los Angeles). Cet imaginaire mosaïque se retrouve encore dans nombre de médias : pour exemple les choix iconographiques du numéro spécial de Sciences et vie, «Climat, comment les villes se préparent aux changements» (décembre 2009) ${ }^{12}$ qui propose un édifiant ensemble de portraits textuels et visuels de toutes les grandes métropoles internationales.

La littérature française a récemment porté un regard plus abstrait et muet sur ces espaces métropolitains : ainsi dans les ouvrages de Jean Echenoz ou d'Éric Laurent on voit des personnages les traverser, s'y déplacer, allant d'espaces publics en espaces publics, anonymement, résumant les métropoles en territoires dont il faut s'émanciper dans le mouvement.

Le projet métropolitain de Paris suscite d'autres approches plus artistiques, plus sensibles, tentant de dépasser les poncifs de la Ville Musée et de la Ville palimpseste ${ }^{13}$. Des photographes ${ }^{14}$ et des plasticiens ${ }^{15}$ arpentent les périphéries, les marges, les confins et les vacances de ce territoire métropolitain, encore indéfini ; via leur propositions visuelles ou leurs interventions (œuvres issues de médiations avec les habitants), ils tentent de donner à tous ces bris et ces zones une densité poétique, d'en transformer la perception commune, d'y tisser des itinéraires insolites, d'y 
capter des mémoires enfouies. Ils veulent saisir une métropole qui ne se dit ni ne se voit pas nécessairement, d'une métropole bien éloignée du design d'ambiance et de la standardisation de son espace public, souvent imposés par l'imaginaire de la mondialisation.

Ce mouvement artistique de reterritorialisation semble s'amplifier, d'autant plus qu'il est parfois accompagné par la puissance publique. Il permet de sortir à la fois des visions déterritorialisantes des Villes « Monde» (pour lesquelles le réseau mondialisé serait plus important que l'environnement urbain « ordinaire ») et des visions apocalyptiques des Mégalopoles (où la pauvreté et l'exclusion seraient les règles, où le désastre ferait lieu d'esthétique). Ces artistes proposent une écriture « en épaisseur », éloignée de la fascination de la fluidité et de l'illimité, invitant à une ou plutôt des lectures du territoire en devenir : ils constituent des inventaires des paysages métropolitains, pointent des différences et des ressemblances entre les lieux, recréent de la limite poétique, donnent aux mots des habitants une résonance singulière et parfois prophétique, leur souci étant de penser une métropole habitable et appropriée au vivre ensemble ${ }^{15}$.

\section{Vers des formes de participation et de concer- tation inédites?}

La concertation est une autre occasion d'entendre la parole et les récits des habitants. Le site «Mon Grand Paris » (www.mongrandparis.fr) s'était présenté, lors de son lancement à la fin 2009 à grand renfort de publicité, comme « le vrai site du débat citoyen » et ceci à la veille du passage du projet de loi à l'Assemblée Nationale. Beaucoup ne virent là qu'une fausse consultation citoyenne ainsi qu'une inversion du processus : une consultation des habitants n'aurait-elle pas du précéder la consultation des architectes?

Cette question de la consultation et de la concertation métropolitaines n'en reste pas moins ouverte. Mais les réponses tardent tant pour des raisons institutionnelles (comment organiser la concertation sans gouvernance effective ?), méthodologiques (quels dispositifs spécifiques pour articuler cette diversité d'échelles et de territoire ?) qu'anthropologiques (comment informer et permettre une parole concrète et contextualisée des habitants sur la métropolisation ?). Recueillir la parole des habitants sur le projet métropolitain suppose que ceux-ci se sentent concernés et en capacité d'émettre une opinion. Les micro-trottoirs réalisés, les recueils de témoignages mis en ligne, ne suffisent pas pour faire des habitants des parties prenantes ayant prise sur le projet. Les sciences de la participation ont un chantier à déployer pour faire émerger une conscience élargie du processus métropolitain. Les études argumentatives et narratologiques ne viendront qu'après pour analyser les paroles et les récits engagés et non plus subis des métropolitains, produits au fil de ces temps de concertation.

Habiter la métropole ce sera de plus en plus la commenter, la raconter, la faire partager, la traduire en témoignages ou en légendes, la peupler de doutes et de certitudes : tout comme le bâti, le récit participe à remodeler la ville. Mais ce récit (individuel ou collectif) ne peut plus être privé ou strictement localisé ${ }^{16}$. Il doit être relayé par une diversité d'intermédiaires (les architectes, les urbanistes, les artistes mais aussi le monde 
associatif) et se publiciser notamment par le biais du réseau Internet, entrer en relation avec d'autres récits, qu'ils soient concordants ou discordants.

\section{Les cultures médiatiques et numériques au service de la métropolisation}

Le récit ordinaire métropolitain se tisse donc, au fil d'une diversité de sources qui souvent s'ignorent : il s'agit encore de bruissements, de traces tant sa construction, sa médiation et sa médiatisation restent timides. Mais il semble évident que les supports numériques vont jouer un rôle central dans sa structuration : une métropole en devenir n'est pas qu'un théâtre, c'est avant tout un espace relationnel et d'expressions anonymes.

\section{Médias localisés, médias alternatifs, cartogra- phies personnalisées}

Si sur Flikr ou Panoramio, la mosaïque de regards amateurs sur Paris, sur ses marges et ses banlieues illustre une lecture bien hésitante de la métropole parisienne, certains sites dédiés à la vie des gens en métropole, au voisinage ou aux parcours métropolitains, affirment depuis deux à trois ans une prise en compte de l'échelle métropolitaine. On peut ainsi évoquer : la ville des gens (www.des-gens.net), Peuplade (www. peuplade.fr), Parisiens $d u$ bout $d u$ monde (http ://www.parisiensduboutdumonde.fr), Gambetta Village (www.gambetta-village.com), ouverts sur l'Est parisien ${ }^{17}$.

Des regards sur la métropole s'y dessinent, appelant de la part de la puissance publique une veille soutenue et continue pour y saisir et décoder ce récit métropolitain en structuration : ainsi y voit-on s'affirmer les figures renouvelées et hybrides de l'habitant et du visiteur (tous les deux piétons, vélocipédistes, passagers du métro et du RER), les emblèmes tangibles de ce territoire métropolitain qui existe bien dans les usages mais ne s'énonce encore que de manière diffuse (le dépassement du périphérique, le Vélib et le tramway, la carte navigo et toutes les formes de cartes et d'itinéraires possibles, le Mac Val et le Stade de France) et s'y dire les manières de pratiquer la métropole.

Internet installe une forme d'espace public, ouvert aux contributions et aux échanges directs. Mais cela ne va pas sans un éclatement énonciatif et une fragmentation narrative : avec Internet, chacun aura son mot à dire, immédiatement et en continu, sur les actions et travaux traduisant au quotidien la métropolisation; l'identité narrative de la métropole s'agencera donc au fil de cette effervescence, en partie aussi via les médias de géolocalisation, les cartographies personnelles et les " automédias urbains » qui interviennent déjà dans la fabrique métropolitaine ${ }^{18}$. Formant un ensemble de contenus, d'explications et de commentaires visant à se saisir de la métropole comme d'un territoire physique à « augmenter ", ceux-ci enrichiront les espaces et les lieux composant une forme inédite de récit éclaté.

\section{Les appels à projets métropolitains : l'interpella- tion des acteurs locaux à agir la métropole}

La délégation Paris Métropole semble avoir pris la mesure de l'apport du numérique au processus de métropolisation en encourageant les initiatives numériques pouvant valoriser le territoire métropolitain, ses espaces et ses temps, ses lieux et 
ses rythmes. Elle a donc lancé à l'automne 2009 l'appel à idées et à projets « Réflexion stratégique et créative sur la Métropolisation de Paris sur le numérique $\gg^{19}$ qui voulait "développer une réflexion sur le rôle des outils numériques dans la construction de la Métropole parisienne mais aussi accompagner et aider au déplacement $d u$ mode de gouvernance non institutionnel qui se met en place à l'échelle de la métropole parisienne » ainsi que "faire avancer la réflexion sur les formes et modes de relations qui peuvent entretenir les collectivités de la Métropole avec leurs habitants en utilisant notamment le mobile et la diversité des médias numériques dans l'espace urbain et métropolitain $»$. Les propositions reçues offrent des pistes intéressantes. Nombre de projets confortent un récit métropolitain territorialisé, le documentent, rendent compte de ses évolutions et mutations, de ses dimensions insolites. Citons ainsi les propositions : Dring 93, Babel Door, Paris Métropole Autrement mais aussi Padim (La Ville des gens), Nous Paris, Métropolitaine culture. Chacun utiliseles médias numériques, en vue de permettre un meilleur rapport de la population à la métropole et en particulier " celle qui est le plus éloignée de la culture et des supports numériques » (personnes isolées socialement et technologiquement).

La délégation semble avoir compris combien le récit métropolitain passe aussi par ces intermédiaires qui relaient les visions et les attentes du terrain. Elle ouvre aussi une réflexion sur les dispositifs permettant d'entrer en relation ou de créer la relation sur un territoire élargi et complexe.

De même, l'appel aux 110 initiatives lancé par le Syndicat Paris Métropole, se veut une démarche pilote (influencée notamment par l'exemple des IBA et du Grand Lyon) pour «faire métropole». Cet appel a vocation de faire émerger « une nouvelle génération de projets métropolitains » "puisant dans l'histoire et révélant la géographie » et pouvant porter tant sur l'urbanisme, le logement, les transports, l'innovation, la culture, le développement que sur les solidarités. La démarche vise à former une coopérative où chaque collectivité compte pour une voix mais aussi «apporte et trouve les ressources pour donner une dimension métropolitaine à ses actions ». Chaque collectivité, chaque groupement de collectivités est invité à présenter une initiative issue soit de leurs services, soit des propositions d'habitants et d'acteurs de leurs territoires ou dont l'action porte sur le territoire.

Ces initiatives, qui pourront être labellisées - le label fonctionnant comme un marqueur symbolique n'ouvrant pas de droit à des financements spécifiques - ont « valeur d'expérimentation » : elles sont énoncées comme "révélatrices $d u$ dynamisme de la métropole » et comme devant "être débattues publiquement ». Leur suivi, l'étude de leur mise en œuvre, de leur appropriation, du jeu d'allers retours entre le niveau local et le niveau métropolitain qu'elles susciteront, permettront de tester la capacité effective des habitants à agir les espaces et les temps de la métropole parisienne.

\section{Vers une identité narrative métropolitaine?}

Au terme de ce parcours, il semble qu'au delà d'un affrontement pour l'hégémonie du sens, ces trois récits se complètent plus qu'ils ne s'excluent. C'est de leur mise en tension qu'un 
imaginaire métropolitain naîtra véritablement, qu'une identité métropolitaine plurielle pourra s'énoncer au croisement du politique, du géopolitique et du poétique.

Le récit permet, par sa temporalité et par son souci de cohérence, de sortir des imageries simplificatrices. Sa structuration, au fil d'une succession de fragments qui se tiennent, la circulation complexe de ses contenus, ses appropriations contradictoires sont nécessaires à l'émergence d'une identité narrative métropolitaine. Celle-ci ne peut se résumer en une voix tout comme elle ne peut être saisie dans son intégralité mais elle s'alimentera jour après jour à cette multitude de paroles et de sources relayées par une diversité de médias et de médiations.

Tout comme la ville de Paris s'est constituée dans le temps au fil de récits pluriels, la métropole n'existera que par ce patient travail de sédimentation, par ces récits fragmentaires qui se tiennent sans être orchestrés par qui que ce soit mais qui font naître un ensemble que certains appellent « macro-récit », dont on peut saisir quelques fils mais qui devient insaisissable à l'analyse, tant sa propagation médiatique et la circulation de ses contenus si divers échappent à la saisie.

Ainsi les instances qui participent à sa fabrication devront prendre en compte les discours ordinaires : que ce soit la Délégation, le Syndicat Paris Métropole, la Société du Grand Paris ou encore l'AIGP, chacun de ces acteurs participera au récit métropolitain sans en avoir le monopole. Il leur faudra envisager la parole et l'expérience des habitants mais en inventant de nouveaux dispositifs qui ne se contentent pas de capter des bris de lan- gage et de les restituer tels quels mais qui visent à faire advenir un sens commun. Une ville Capitale et encore plus une métropole mondiale doit s'inventer en permanence : l'imaginaire devient non seulement une ressource mais un levier de métropolisation : il approfondit le territoire, lui donne de l'épaisseur, permet d'en affronter la mémoire et les métamorphoses, d'en ouvrir les possibles et d'en multiplier les interprétations et lectures. 
$\mathrm{N} \cdot \mathrm{O} \cdot \mathrm{T} \cdot \mathrm{E} \cdot \mathrm{S}$

1. Voir notamment mes précédents articles : « L'inscription du projet de la Métropole de Paris dans l'espace public numérique : émergences et ouvertures ", in Quaderni, n71, Hiver 2009/2010 ; «Les territoires numériques : au-delà de l'information localisée, l'hospitalité au fil des écrans », in Quaderni, $\mathrm{n}^{\circ} 72$, printemps 2010 .

2. Voir Alain Bourdin, «La Métropole des individus », Paris, Éditions de l'Aube, 2008.

3. Voir 1'article d'Annick Bélanger "Montréal vernaculaire, Montréal spectaculaire : dialectique de l'imaginaire urbain », in revue Sociologie et Sociétés, vol.37, n¹, 2005, p.13/34 ( http ://id.erudit.org/ iderudit/012274ar).

4. Dès 1983, François Mitterrand évoqua la possibilité politique et urbanistique d'un Grand Paris ; le projet de « Banlieues 89 » puis celui de l'association « Paris $75021 »$ furent des creusets essentiels où émergèrent les principales thématiques de Paris Métropole.

5. Ainsi en 2009, la Petite synthèse de la Consultation Grand Paris.

6. Ce syndicat s'attache à construire " un dialogue pluraliste entre les communes, les intercommunalités, les départements, la région, sur le devenir de la Métropole ». Il pourrait préfigurer selon certains une future assemblée métropolitaine, comme le sont le Grand Londres ou le Grand New York.

7. Lire la déclaration de Nicolas Sarkozy à la revue AA, entretien de février 2010 : «il me semble important de conduire tous les projets ensemble de front. Il faut que les 1000 projets s'épanouissent ». Voir le document publié au printemps 2010 par le Syndicat « 110 initiatives pour la Métropole », réalisé avec la collaboration de l'APUR et de l'IAU.

8. 10 équipes autour de 10 architectes de renom et donc
10 desseins d'aménagement pour adapter la métropole parisienne à l'ère de l'après Kyoto : Antoine Grumbach ; Jean Nouvel ; Djamel Klouche ; Roland Castro ; Christian De Portzamparc ; Yves Lion ; Rogers Stirk Harbour et partners ; Bernardo Secchi et Paola Vigano ; Wini Maas ; Finn Geipel et Guilia Andi.

9. Voir la revue d'architecture AA qui a demandé en février au Président et aux 10 équipes de faire le point sur l'avenir de la Région Capitale et aux architectes d'actualiser, de synthétiser et de critiquer leurs propositions au vu des mois passés.

10. Voir l'article « The Vernacular mode, locating the non institutional on the practice of citizenship » de Robert Glenn Howard, in Public modalities : rhetoric, culture, media and the shape of public life, Daniel C. Browser et Robert Asen ( directeurs ), Editions Alabama, 2010.

11. Soutenus par le fondateur, directeur de publication et de la rédaction des Inrockuptibles, par le directeur de la Fondation Lagardère et une agence de création.

12. Revue Sciences et Vie, "Questions Réponses, Spécial Sommet de Copenhague », décembre 2009.

13. Voir notamment le $n^{\circ} 12$ de la revue Stradda : « Grand Paris, quelle place pour les artistes? ».

14. Dès 1990 des créateurs abordèrent la question métropolitaine : ainsi le texte Les passagers $d u$ Roissy Express de François Maspero, accompagné des photographies d'Anaïk Frantz qui capte les paysages et les humeurs de la métropole en prenant la ligne $\mathrm{B}$ du RER. Plus récemment, on peut se référer à l'exposition « No Limit» et à son catalogue (Pavillon de L'Arsenal, décembre 2008), réalisés à partir d'une étude prospective de l'insertion urbaine des périphéries de Paris.

15. Voir les travaux de Malt Martin (www.atelier-malt. martin.net et www.agrafmobile.net), Claire Pétetin et son travail artistique avec l'agence « Le troisième pôle » sur le projet Ville + . 
16. Le catalogue de la Biennale de Lyon (septembre 2009/janvier 2010) portant sur Le spectacle au quotidien permet de prolonger cette réflexion en actualisant l'idée de communautés imaginées à l'heure de la métropolisation et de la mondialisation.

17. Ou encore, dédiés à la visite et au tourisme mais restant encore très parisiens : «Paris par rues méconnues » (www.paris-prm.com), Paris Go (www.parisgo. fr), « Paris bouge » (www.parisbouge.com), « Paris nightlife » (www.parisnightlife.fr) ou « Parisien d'un jour » (www.parisiendunjour.com).

18. Ainsi les projets accompagnés par Cap Digital et notamment « Terra Numerica ».

19. Appel que nous avons initié et lancé avec la Délégation (entre juin 2009 et avril 2010) .

\section{$R \cdot E ́ \cdot S \cdot U \cdot M \cdot E ́$}

Pour exister et se déployer tant mondialement que localement, les métropoles font l'objet de mises en récit dont la nature et les auteurs, les thématiques et les logiques éditoriales se renouvellent et se diversifient. Ainsi, le processus de la métropolisation de Paris s'accomplit au fil d'un faisceau complexe d'écritures enchevêtrées : les stratégies narratives en place, notamment celles de «Paris Métropole» et du " Grand Paris », actualisent des visions et des projets à la fois antagonistes et complémentaires. De l'écriture experte du projet de la Mairie de Paris au récit épique de Nicolas Sarkozy, un imaginaire politique et urbanistique métropolitain s'est ébauché mais il s'enrichit aujourd'hui des productions et des regards d'une diversité croissante d'énonciateurs, politiques, médiatiques et artistiques mais aussi, certes de manière encore éparse, anonymes ( de l'habitant au touriste).

\section{Summary}

To exist and be contextualized as global and local, cities are being put into a narrative whose nature and whose authors, themes and editorial logics grow and diversify. Thus, the metropolization process of Paris transpires over a complex set of interlocking entries. The narrative strategies in place update visions and projects that are antagonistic and complementary, including those of Paris Métropole and Grand Paris. From expert analysis of the project of the City of Paris to the epic discourse of Nicolas Sarkozy, a political imaginary and urban metropolitan identity have been formed. Today, this imaginary and identity have enriched productions and views on an expanding variety of enunciators representing political, media and art milieus, but also those who are anonymous (from the local citizen to tourists). 